\title{
A Comparison of the Korean and Japanese Medical Technician's. Etc. Act Systems Focusing on Physical and Occupational Therapists
}

Tae-Hyung Yoon

Department of Occupational Therapy, Division of Health Science, Dongseo University, Center for Radiological Environment and Health Science, Community-based Arts for Rehabilitation \& Education Laboratory, Busan, Korea

Purpose: The aim of this study was to compare the "Physical Therapist and Occupational Therapist Act" of Japan and the "Medical Technician's. Etc. Act" of Korea in order to establish the grounds for improvement of effective law-making.

Methods: We obtained the "Medical Technician's. Etc. Act" from the Korean Ministry of Government Legislation and the "Physical Therapist and Occupational Therapist Act" from the Japanese Ministry of Health, Labor and Welfare. It was translated from an association by related Japanese and experts.

Results: The laws consisted of acts, enforcement ordinances, and enforcement regulations in both Korea and Japan. In the Korean case, eight occupations were defined in one law including six types of medical technicians, as well as medical recorders and opticians. The "Physical Therapist and Occupational Therapist Act" in Japan is composed of 6 chapters and 22 articles, while the Korean Act consists of 33 articles without chapters. Among them, 11 articles covered the establishment and management of dental laboratories and eyeglass shops, and only 22 articles were related to physical therapists and occupational therapists.

Conclusion: Independent laws should be established for each type of medical technician. They must be comparable to Japanese laws on physical therapists and occupational therapists as well as clinical pathologists, dental hygienists, dental technicians, radiologic technologists, medical recorders, and opticians.

Keywords: Medical technician's. Etc. Act, Occupational therapist, Physical therapist

\section{서 론}

한국은 의료보조원법을 1963 년에, 의료기사법을 1973 년에 제정하여 물리치료사의 지위를 마련하여 큰 변화 없이 현재에 이르고 있다. ${ }^{1}$ 모 든 보건의료정책 결정은 관련법과 서로 다른 보건의료인의 이익집단 의 갈등 구조 속에서 발전되어 왔다고 해도 과언이 아니다. 그러나 그 동안 이에 대한 관련 연구가 부족하였으며 국가 간 관련법률의 비교 는 전무하였다. 현재 우리나라의 보건의료인에 대한 면허와 규정은 '의료법과 '의료기사 등에 관한 법률'에서 규정하고 있다.,3 '의료법'에 서는 의료인인 의사, 치과의사, 한의사, 조산사 및 간호사를 규정하고 있으며, '의료기사 등에 관한 법률에서는 의료기사와 의무기록사 및 안경사의 자격.면허 등에 관한 필요한 사항을 정하고 있다. ${ }^{5}$ 동법에서

Received Mar 22, 2016 Revised Apr 28, 2016

Accepted Apr 29, 2016

Corresponding author Tae-Hyung Yoon

E-mail yth@gdsu.dongseo.ac.kr
의료기사라 함은 임상병리사, 방사선사, 물리치료사, 작업치료사, 치 과기공사 및 치과위생사이다. 게다가 이 법에서는 의무기록사와 안경 사까지 포함하여 다루고 있다. 최근 안경사의 단독법 제정 추진으로 의료계와 안경사 협회가 마찰을 보이고 있으며, 이어서 물리치료사 와 작업치료사가 포함된 30 만 재활치료사가 단독법 제정 및 단독 개 업을 주장하여 이 역시 의료계와 심각한 대립각을 세우고 있다. ${ }^{6}$ 이 러한 주장과 갈등은 의료의 영역이 약물투여나 수술과 같은 급성기 질환에서 만성질환을 포함한 정서 및 신체장애를 가진 장애인을 대 상으로 하는 재활, 보건의료서비스 및 복지분야로 확대되면서 계속 적으로 증가할 것으로 예상된다. 최근 의료기사들의 단독개업은 차 치하고라도 단독법의 제정을 통해 해당 전문인력의 질적 향상을 가 져와야 한다는 주장이 제기되고 있다. 왜냐하면 해당전문인력들의

Copylight (C2016 The Korea Society of Physical Therapy

This is an Open Access article distribute under the terms of the Creative Commons Attribution Non-commercial License (Http:// creativecommons.org/license/by-nc/3.o.) which permits unrestricted non-commercial use, distribution, and reproduction in any medium, provided the original work is properly cited. 
질적 향상이 곧 국민의 건강을 향상시키는 일이기 때문이라는 주장 이다.

보다 구체적으로 살펴보면, $\mathrm{OECD}$ 국가 중 한국은 물리치료사와 작업치료사의 단독개업이 금지되어 있고 이는 세계적 추세에 역행하 는 일이라 할 수 있으며 이러한 체계로는 재활치료분야의 발전을 더 이상 기대하기 어렵다. 재활선진국이라고 할 수 있는 일본에서도 단 독개업이 금지되어 있기는 하지만 물리치료사와 작업치료사법이 단 독으로 존재하여 면허와 그 역할에 대해서 상세히 규정하고 있다. ${ }^{8}$ 일 본의 의료법에 나타난 의료인의 개념은 '의사, 치과의사, 약제사, 간호 사, 기타 의료를 담당하는 자"라고 모든 보건의료인을 포함하고 있으 며 기타의료를 담당하는자에는 보건사, 조산사, 치과기공사, 치과위 생사, 시능훈련사, 임상검사기사, 위생검사기사, 이학요법사(물리치료 사), 작업치료사, 진료방사선기사, 임상공학기사, 의지장구사, 응급구 조사, 안마.마사지.지압사, 침사, 구사, 유도종복사가 있고 각각의 독 립된 개별법이 존재한다. 또한 그 역사를 살펴보면 일본은 1907 년 이 학요법사 및 작업요법사법이 제정되어 100 년의 역사를 거쳐 개정을 통해 현재의 법이 마련되었으나, 한국은 1973년 “의료기사 등에 관한 법률”이 제정되어 이에 비해 그 역사가 짧다고 할 수 있다.

이에 본 연구에서는 여러 의료기사 중 최근 이슈가 되고 있는 물리 치료사와 작업치료사를 선정하여 한국과 법 체계가 가장 비슷한 일 본의 “이학요법사 (물리치료사)와 작업치료사법”과 한국의 “의료기 사 등에 관한 법률”의 체계와 내용을 중심으로 비교함으로써 문제점 을 살펴보고 효율적인 법률의 개선방향을 도출할 목적으로 시도되 었으며 그 구체적인 목표는 다음과 같다.

첫째, 일본의 물리치료사와작업치료사법에 해당되는 "이학요법사 (물리치료사) 및 작업치료사법”을 분석한다.

둘째, 한국의 “의료기사 등에 관한 법률”을 분석한다.

셋째, 일본과 한국의 법률을 비교. 분석하고 개선방향을 도출한다. 본 연구에서는 우리나라와 가장 보건의료 관련법 체계가 비슷한 일본의 의료기사법 (이학요법사 및 작업요법사법)을 중심으로 두 나 라 의료기사법의 구성체계, 특히 의료기사법에 담아야 할 기본 원칙 과 법 (법률)-시행령-시행규칙으로 정해진 규정을 중심으로 한국과 일본 의료기사 등에 관한 법률의 체계를 비교 분석하였다. 이를 위해 우리나라 법제처에서 “의료기사 등에 관한 법률”, 시행령, 시행규칙에 대해 자료를 취합. 분석하였고 일본의 후생노동성을 통해 “이학요법 사 (물리치료사) 및 작업요법사법”을 조사했다. “이학요법사 (물리치 료사) 및 작업요법사법”의 전문을 일본 국제의료복지대학 박사과정 생에게 1 차 번역을 의뢰한 후 서울 마포구에 소재한 관련기관 $(000$ 학 회내 노인연구정보센터)을 통해 최종 번역하였다. 또한, 매 번역마다 법률 자문교수에게 자문을 받았다.

\section{본 론}

\section{1. 일본의 “이학요법사 (물리치료사) 및 작업치료사법”의 체계}

1) 법의 개요

일본의 “이학요법사 (물리치료사) 및 작업치료사법”도 한국과 같이 법률, 시행령, 시행규칙으로 나뉘어져 있으며 그 내용은 Table 1과 같 다. 모두 6장 22 조로된 법률과 부칙으로 구성되어 있었다. 총 6 장의 내 용은 제 1 장 총칙, 제 2 장 면허, 제 3 장 시험, 제 4 장 업무, 제 5 장 물리치료 사와작업치료사시험위원, 제6장 벌칙으로 구성되어 있었다. 8

\section{2) 법의 주요 내용}

(1) 제1장 총칙 (제1조-제2조)

제 1 장 총칙에서는 물리치료사 및 작업치료사의 자격과 업무가 적정 하게 운용되도록 한다는 목적을 규정하였고 제 2 장 정의에서는 물리 치료와작업치료의 정의를 나타내었다.

\section{(2) 제2장 면허 (제3조-제8조)}

제 3 조에서는 물리치료사와 작업치료사의 면허 획득을 위해 국가시 험에 합격해야 함을 제 4 조에서는 면허를 줄 수 없는 자에 해당하는 규정을 정하고 있다. 또한 제 5 조에서는 후생노동성에 물리치료사와 작업치료사 명부를 비치하여 면허에 관한 사항을 등록할 수 있도록 하였으며 제 6 조에서는 등록 및 면허증의 교부에 대하여 정하고 있다. 특이한 점은 제6조 2 에서 면허 신청자에 대해 어떠한 이유로 면허를 주지 않게 될 때 해당자를 배려하여 사전에 해당 신청자에게 그 취지 를 통지하고, 그 신청자의 요구가 있을 때에는 후생노동성장관이 지 정하는 직원에게 그 의견을 청취하도록 하고 있다. 제7조에서는 면허 의 취소와 재면허 교부를 정하고 있으며 모든 일을 처리할 때 심의 위 원회를 두어 의견을 듣도록 하고 있었다. 제 8 조에서는 이외의 모든 면 허와 관련된 사항은 시행령으로 정하고 있었다.

\section{(3) 제3장 시험 (제9조-제14조)}

제 9 조에서는 국가시험의 목적에 관한 것으로 물리치료사 또는 작업 치료사로서 필요한 지식 및 기능에 대해 실시한다고 규정하였다. 제 10 조에서는 국가시험을 매년 적어도 1 회, 후생노동성장관이 실시한 다고 되어 있다. 제 11 조와 제 12 조에서는 물리치료사와작업치료사 국 가시험의 수험자격에 대해서 논하고 있는데, 각 학과에서 3 년 이상 물리치료나 작업치료사로서 필요한 지식 및 기능을 습득한 자가 시 험을 볼 수 있으며, 특이한 점은 물리치료사 면허자가 후생노동성이 인정한 물리치료 양성시설에서 2년 이상 물리치료에 관한 지식 및 기 능을 습득한 자는 작업치료사 시험을, 작업치료사 면허자가 해당 양 성시설에서 2 년 이상 작업치료에 관한 지식 및 기능을 습득한 자는 
Table 1. System of the "Physical Therapist and Occupational Therapist ACT" in Japan

\begin{tabular}{|c|c|c|c|c|}
\hline & & $\mathrm{ACT}$ & Enforcement Ordinance & Enforcement Regulations \\
\hline \multirow{2}{*}{$\begin{array}{l}\text { Chapter } 1 \text { General } \\
\text { Provision }\end{array}$} & Article 1 & Purpose & & \\
\hline & Article 2 & Definitions & & \\
\hline \multirow[t]{7}{*}{ Chapter 2 License } & Article 3 & License & Request License & $\begin{array}{l}\text { - Prohibition License } \\
\text { - Consideration of treatment } \\
\text { - Procedure and Request License }\end{array}$ \\
\hline & Article 4 & Disqualifications & & \\
\hline & Article 5 & $\begin{array}{l}\text { List of Physical Therapist and Occupational } \\
\text { Therapist }\end{array}$ & $\begin{array}{l}\text { Registration of List } \\
\text { Correct List }\end{array}$ & $\begin{array}{l}\text { - Registration of List } \\
\text { - Request of Correct List }\end{array}$ \\
\hline & Article 6 & Registration and Issue of the License & $\begin{array}{l}\text { - Deletion of registration } \\
\text { - Renewal and Issue of License } \\
\text { - Reissue of License } \\
\text { - Return of License }\end{array}$ & $\begin{array}{l}\text { - Form a License } \\
\text { - Issue Application of License } \\
\text { Renewal } \\
\text { - Application of License Reissue } \\
\text { - Payment of the Registration of } \\
\text { License Tax and Fee }\end{array}$ \\
\hline & Article 6-2 & Listening to Opinion & & \\
\hline & Article 7 & Revocation of the License & & \\
\hline & Article 8 & Delegation to government decree & - Delegation to government & \\
\hline \multirow{7}{*}{ Chapter 3 Examination } & Article 9 & Purpose of the Examination & & \\
\hline & Article 10 & Conduct an Examination & & $\begin{array}{l}\text { - Examination subject } \\
\text { - Notice of Exam date } \\
\text { - Application of Identification } \\
\text { - Issue of Certificate } \\
\text { - Issue of Certificate and Fee } \\
\text { - Payment Fee }\end{array}$ \\
\hline & Article 11 & $\begin{array}{l}\text { Qualifications of candidacy for Physical therapist } \\
\text { Examination }\end{array}$ & $\begin{array}{l}\text { - Designation of University or Facilities } \\
\text { - Request for designation } \\
\text { - Approval (Declaration) of Alteration } \\
\text { - Report } \\
\text { - Collection (Instruction) of Report } \\
\text { - Appointment cancellation } \\
\text { - Request Cancellation of Appointment } \\
\text { - Special Case of University or Facility by } \\
\text { Government }\end{array}$ & \\
\hline & Article 12 & $\begin{array}{l}\text { Qualifications of candidacy for Occupational } \\
\text { therapist Examination }\end{array}$ & & \\
\hline & Article $12-2$ & Consultation to Advice Council & & \\
\hline & Article 13 & Prohibition to illegality & & \\
\hline & Article 14 & Delegation to government decree & $\begin{array}{l}\text { - Delegation to government decree } \\
\text { - Competent Minister etc. }\end{array}$ & \\
\hline \multirow[t]{4}{*}{ Chapter 4 Duty } & Article 15 & Duty & & \\
\hline & Article 16 & Duty of Confidence & & \\
\hline & Article 17 & Restriction of the PT and OT & & \\
\hline & Article 17-2 & Delegation to Authority & & \\
\hline \multirow{2}{*}{$\begin{array}{l}\text { Chapter } 5 \text { Examination } \\
\text { Board for PT and OT }\end{array}$} & Article 18 & Examination board for PT and OT & - Examination board for PT and OT & \\
\hline & Article 19 & $\begin{array}{l}\text { Prohibition of Illegality for the Exam Person in } \\
\text { Charge }\end{array}$ & $\begin{array}{l}\text { - Classification Business } \\
\text { - Delegation to Authority }\end{array}$ & \\
\hline \multirow[t]{3}{*}{ Chapter 6 Penalty } & Article 20 & $\begin{array}{l}\text { Leakage of test question/illegal score:Less than a } \\
\text { Year in Jail, Fine of 500,000 yen }\end{array}$ & & \\
\hline & Article 21 & Penalty of the Divulgence & & \\
\hline & Article 22 & Penalty of the name of the PT and OT & & \\
\hline \multirow[t]{6}{*}{ Supplementary Provision } & & Special Acquisition of the License & & - Special Examination Subject \\
\hline & & $\begin{array}{l}\text { Special Acquisition of the qualifications of candidacy } \\
\text { for examination }\end{array}$ & & $\begin{array}{l}\text { - More than Secondary School } \\
\text { Graduate }\end{array}$ \\
\hline & & $\begin{array}{l}\text { Interim Measures of Unfavorable Disposition on } \\
\text { Consultation }\end{array}$ & & \\
\hline & & Interim Measures of Penalty & & \\
\hline & & Interim Measures of Hearing & & \\
\hline & & Delegation to government decree & & \\
\hline
\end{tabular}

물리치료사 시험을 볼 수 있는 자격이 주어졌다. 또한 외국의 물리치 료나작업치료학과를 졸업했거나, 외국 물리치료사나작업치료사 면 허 소지자는 해당 국가시험을 볼 수 있는 자격이 주어진다. 제 12 조의 2 에서는 해당 국가시험의 과목 또는 실시 혹은 합격자 결정의 방법을
정하려고 할 때에는 사전에 의료심의회 (자문위원회)의 의견을 들어 야 한다. 제 13 조에서는 부정행위에 관계 있는 자에 대하여 그 수험을 정지시키거나 무효로 할 수 있으며 이 경우에 또한 그 자에 대하여 기 간을 정해 해당 국가시험의 응시를 허락하지 않을 수 있다. 제 14 조에 
서는 이 법률에서 규정한 것 외의 학교 또는 물리치료사나 작업치료 사양성시설의 지정에 대해서 시행령으로 정하고 있다.

\section{(4) 제4장 업무 등 (제15조-제17조)}

제 15 조에서는 물리치료사와 작업치료사의 업무에 관해서 규정하고 있다. 제 16 조에서는 업무상 알게 된 자의 비밀을 누설하지 못하는 비 밀보장의 의무에 대해 규정해 놓고 있다. 제 17 조에서는 해당 면허 소 지자가 아닌 경우 물리치료사 또는 기능치료사, 작업치료사 또는 직 능치료사라는 명칭을 사용하지 못하도록 하고 있다. 제17조의 2에서 는 후생노동성장관의 권한을 지방후생국장에게 위임할 수 있도록 하였다.

\section{(5) 제5장 물리치료사 (작업치료사) 시험위원 (제18조-제19조)}

제 18 조에서는 해당 국가시험에 관한 사무를 주관하기 위해서 후생노 동성에 물리치료사와작업치료사 시험위원을 두게 하고 필요한 사항 은 시행령으로 정하도록 하고 있다. 제 19 조에서는 해당 시험위원 외 의 국가시험에 관한 사무를 주관하는 자는 그 사무의 시행에 있어서 규칙을 준수하고 부정행위가 없도록 규정하고 있다.

\section{(6) 제6장 벌칙 (제20조-제22조)}

제 20 조에서는 고의로 또는 중대한 과실에 의해 사전에 시험문제를 누출하거나 고의로 부정 채점을 한 자에 대해 1년 이하의 징역 또는 50 만 엔 이하의 벌금에 처하고 있다. 제 21 조에서는 비밀보장의 의무 를 위반한 자에 대해 50 만 엔 이하의 벌금을 정하고 있으며 모든 죄는 고소가 없으면 공소를 제기할 수 없다. 제22조는 물리치료사 또는 작 업치료사 명칭의 사용정지를 명령받은 자로서 해당 정지를 명령 받 은 기간 중에 물리치료사 또는 작업치료사의 명칭을 사용한 자에 대 해서 30 만 엔 이하의 벌금에 처하고 있다. 부칙에서 특이할 만한 점은 면허의 특례인데, 외국에서 물리치료사 또는 작업치료사 면허에 상 당하는 면허를 받은 자로서 필요한 지식 및 기능을 가지고 있다고 인 정한 자에 대해서는 면허취득 여부에 관계없이 당분간 물리치료사 또는 작업치료사의 면허를 줄수 있다고 정하고 있다.

\section{2. 한국의 “의료기사 등에 관한 법률”의 체계}

\section{1) 법률의 개요}

한국의 “의료기사 등에 관한 법률”도 법률, 시행령, 시행규칙으로 나 뉘어져 있으며 그 내용은 Table 2와 같다. “의료법”, “정신보건법”, "장애 인복지법”과 달리 “의료기사 등에 관한 법률”은 법이 아니라 법률로 표현하고 있으며, 장 없이 조로만 구성되어 있다. 총 33 조로된 법률과 부칙으로 구성되어 있었으나 제17조, 18조, 19조가 1999년 2월 28일 삭 제되어 실제적으로는 30 조로 구성되어 있다 할 수 있다. 더구나, 치과
기공소와 안경업소의 개설등록과 관련된 물리치료사와 작업치료사 와 직접 연관 없는 조항 10 여 가지를 제외한다면 총 22 조의 내용으로 구성되어 있다. 이 총 24 조의 내용도 6 종의 의료기사 모두를 아울러 표현하고 있어 실제적인 물리치료사나작업치료사만의 법은 아니다.

\section{2) 법률의 주요내용}

(1) 목적과 정의, 의료기사의 종류 및 업무 범위와 한계 (제 1조-제3조) 제 1 조에서 이 법의 목적인 의료기사, 의무기록사 및 안경사의 자격. 면허 등에 관하여 필요한 사항을 정함으로써 국민의 보건 및 의료 향 상에 이바지함을 목적으로 한다고 정하고 있다. 제 1 조의 2 에서는 6 종 의 의료기사를 한 가지로 구분하여 "의사 또는 치과의사의 지도 아 래 진료나 의화학적 검사에 종사하는 사람"으로 규정하고 있다. 제2 조에서는 의료기사의 종류를 임상병리사, 방사선사, 물리치료사, 작 업치료사, 치과기공사 및 치과위생사 모두 6 종으로 규정하고 있다. 제 3 조에서는 의료기사, 의무기록사 및 안경사의 업무 범위와 한계를 대 통령령으로 정하고 있다. 그 시행령을 살펴보면 물리치료사는 온열치 료, 전기치료, 광선치료, 수치료, 기계 및 기구치료, 마사지·기능훈련. 신체교정운동 및 재활훈련과 이에 필요한 기기.약품의 사용.그 밖의 물리요법적 치료업무로 규정하고 있으며 작업치료사는 신체적·정신 적 기능장애를 원활하게 회복시키기 위해 일상생활에서 사용하는 물체나 기구를 활용한 감각. 활동훈련, 작업적 일상생활훈련, 인지재 활치료, 삼킴장애 재활치료, 상지보조기 제작 및 훈련, 작업수행분석 및 평가업무, 그 밖의 작업요법적 훈련.치료 업무로 규정하고 있다.

\section{(2) 면허취득, 시험 및 응시자격의 제한 (제4조-제7조)}

제 4 조에서는 의료기사가 되기 위해 해당 대학을 졸업한 자와 외국의 해당 의료기사 등의 면허를 받은 사람이 면허를 보건복지부장관으 로부터 받을 수 있다. 제 5 조 결격사유에서는 정신질환자, 마약중독 자, 금치산자, 한정치산자 그리고 금고 이상의 실형 선고받고 그 집행 이 끝나지 아니하거나 면제되지 아니한 사람으로 정하고 있다. 제6조 에서는 국가시험은 해마다 1 회 이상 보건복지부장관이 실시하고 시 험관리 능력이 인정되는 관계전문기관으로 하여금 국가시험을 관리 하게 할 수 있으며 현재 한국보건의료인국가시험원에서 담당하고 있 다. 국가시험의 범위, 국가시험의 시행과 공고, 시험위원, 국가시험의 응시에 관한 사항은 시행령에서 시험과목과 합격자 결정 등에 관한 사항은 시행규칙에서 정하고 있다. 제7조에서는 부정행위와 관련해 서 응시자격의 제한에 대해서 설명하고 있다.

(3) 면허의 등록, 무면허자의 업무금지 및 비밀누설의 금지, 실태 등의 신고 (제8조-제11조)

제 8 조는 면허의 보건복지부대장 등록에 대해서 규정하고 있다. 제 9 
Table 2. System of the "Medical Technician etc ACT" in Korea

\begin{tabular}{|c|c|c|c|c|}
\hline & $\mathrm{ACT}$ & Enforcement Ordinance & Enforcement Regulations & $\begin{array}{c}\text { Article related } \\
\text { to PT or OT }\end{array}$ \\
\hline Article 1 & Purpose & Purpose & Purpose & O \\
\hline Article 1-2 & Definitions & & & 0 \\
\hline Article 2 & Classifications of Medical Technicians & & & 0 \\
\hline Article 3 & Scope and Limit of Duties & $\begin{array}{l}\text { Scope and Limit of Duties (Medical } \\
\text { Technician, Medical Recorder, } \\
\text { Opticians) }\end{array}$ & & 0 \\
\hline Article 4 & License & & & 0 \\
\hline Article 5 & Disqualifications & & & 0 \\
\hline Article 6 & National Examination & $\begin{array}{l}\text { - Scope of the National Exam } \\
\text { - Enforcement Notices of the } \\
\text { National Exam } \\
\text { - Examination Board } \\
\text { - Take the National Exam }\end{array}$ & $\begin{array}{l}\text { - Examination Subject } \\
\text { - Determination of Successful Candidate }\end{array}$ & 0 \\
\hline Article 7 & Restriction, etc. of Examinees' Qualifications & & $\begin{array}{l}\text { - Restriction of the Wrongdoer } \\
\text { - Reconsideration of Regulation }\end{array}$ & 0 \\
\hline Article 8 & Registration, etc. of License & - Issue of the License & $\begin{array}{l}\text { - Register of License } \\
\text { - Issue of the License }\end{array}$ & 0 \\
\hline Article 9 & Prohibition, etc. of Services by Unlicensed Persons & & & 0 \\
\hline Article 10 & Prohibition of Divulgence of Secrets & & & 0 \\
\hline Article 11 & Report on Actual conditions, etc. & $\begin{array}{l}\text { - Report of the Condition } \\
\text { - Build a Reporting System }\end{array}$ & - Report on the condition & O \\
\hline Article 11-2 & $\begin{array}{l}\text { Establishment Registration, etc. of Dental } \\
\text { Laboratories }\end{array}$ & & $\begin{array}{l}\text { - Registration of Dental Laboratories } \\
\text { - Facilities and Equipment of Dental } \\
\text { Laboratories }\end{array}$ & $x$ \\
\hline Article 11-3 & $\begin{array}{l}\text { Compliance Requirements for Dental Technicians, } \\
\text { etc. }\end{array}$ & & $\begin{array}{l}\text { - Request for production of Dental } \\
\text { Technician }\end{array}$ & $x$ \\
\hline Article 12 & Establishment Registration, etc. of Eyeglass Shops & & $\begin{array}{l}\text { - Registration of Eyeglass Shops } \\
\text { - Standard of an Eyeglass Shop }\end{array}$ & $x$ \\
\hline Article 13 & Report on Closure, etc. & & $\begin{array}{l}\text { - Eyeglass Shop registry and registration } \\
\text { certificate } \\
\text { - Report on Closure }\end{array}$ & $\times$ \\
\hline Article 14 & Prohibition of Exaggerated Advertisement, etc. & & & $x$ \\
\hline Article 15 & Reporting, Investigation, etc. & & & $x$ \\
\hline Article 16 & Association & & & 0 \\
\hline Article $17,18,19$ & Deleted & & & \\
\hline Article 20 & Supplementary Education & & $\begin{array}{l}\text { - Supplementary Education } \\
\text { - Submission of Supplementary } \\
\text { Education Program and Result Report } \\
\text { - Method of Supplementary Education } \\
\text { - Preservation documents }\end{array}$ & 0 \\
\hline Article 21 & Revocation, etc. of License & - Revocation of License & $\begin{array}{l}\text { - Request of License Revocation } \\
\text { - Replacing Certificate for License } \\
\text { - Withdrawal of License }\end{array}$ & 0 \\
\hline Article 22 & Suspension of Qualification & - Scope of injury to dignity & & 0 \\
\hline Article 23 & Rectification Order & & & $x$ \\
\hline Article 24 & Revocation, etc. of Establishment Registration & & - Notice of Administrative disposition & $x$ \\
\hline Article 25 & Standards for Administrative Dispositions & & & 0 \\
\hline Article 26 & Hearings & & $\begin{array}{l}\text { - Processing of Sensitive Information and } \\
\text { Identification Information }\end{array}$ & 0 \\
\hline Article $26-2$ & Request of the Data Service & & & $\times$ \\
\hline Article 27 & Fees & & - Fees etc. & 0 \\
\hline Article 28 & Delegation or Entrustment of Authority & - Entrust business & & 0 \\
\hline Article 29 & Relation to other Acts & & & $\times$ \\
\hline Article 30 & Penal Provisions & & & 0 \\
\hline Article 31 & Penal Provisions & & & 0 \\
\hline Article 32 & Joint Penal Provisions & & & 0 \\
\hline Article 33 & Administrative Fines & - Imposition of Fine for Negligence & & 0 \\
\hline
\end{tabular}

조에서는 무면허자의 업무금지에 관한 사항을 설명하고 있고, 제 10 조 에서는 업무상 비밀누설금지에 대한 내용을 담고 있으며, 제11조에서 는 면허 취득 후 3 년마다 실태와 취업상황을 보건복지부장관에게 신 고하여야함을 나타내고 있다.
(4) 치과기공소와 안경업소에 관한 조항 (제11조의 2-제15조) 제 11 조의 2 , 제 11 조의 3 , 제 12 조, 제 13 조, 제 14 조, 제 15 조는 치과 기공소 와 안경업소에 관한 내용으로 치과기공소의 개설등록, 치과기공사 등의 준수사항, 안경업소의 개설등록, 폐업 등의 신고, 과대광고 등의 
금지, 보고와 검사 등에 관한 내용을 담고 있다.

(5) 의료기사협회, 보수교육 (제16조-제20조)

제 16 조는 의료기사가 협회를 설립할 수 있으며 협회는 법인으로 한다 고 규정하고 있다. 제20조는 보수교육에 대하여 설명하고 있으며 보 다 구체적인 시기와 기간 등을 시행규칙에서 정하고 있다.

(6) 면허취소, 자격정지 (제21조-제22조)

제 21 조에서는 면허 취소 사유에 대해 설명하고 있으며 시행령과 시행 규칙에서 면허증의 재발급 및 신청, 면허증을 갈음하는 증서와 면허 증의 회수에 대해 설명하고 있다. 제 22 조에서는 자격의 정지에 대해 설명하고 있으며 시행령에서 의료기사 등의 품위손상행위의 범위에 대해 정하고 있다.

(7) 치과기공소 또는 안경업소의 시정명령, 개설등록의 취소 (제23조-제24조) 제 23 조, 제 24 조는 치과 기공소와 안경업소의 시정명령과 개설등록의 취소에 관한 내용이다.

\section{(8) 행정처분의 기준, 청문 (제25조-제26조)}

제 25 조는 행정처분의 기준에 대해서 제 26 조는 면허의 취소와 등록 의 취소에 따른 청문에 대한 내용을 다루고 있다.

(9) 치과기공소와 안경업소의 자료제공의 요청 (제26조의 2)

제 26 조의 2에서는 치과 기공소와 안경업소의 설치 및 운영 현황에 대 한 자료제공 요청에 대한 내용을 다루고 있다.

(10) 수수료, 권한의 위임 또는 위탁 (제27조-제28조)

제 27 조에서는 면허를 받으려는 사람, 면허증을 재발급받으려는 사람, 국가시험에 응시하려는 사람에 대한 수수료에 대해 나타내고 있으 며, 제 28 조에서는 보건복지부장관이 지방자치단체장이나 보건소장 에게 권한의 위임 또는 위탁에 대해 설명하고 있다.
(11) 안경업소와 다른 법률과의 관계 (제29조)

제29조에서는 안경업소의 등록 및 그 취소 등에 대하여 다른 법률과 의 관계에 대해규정하고 있다.

(12) 벌칙, 양벌규정 및 과태료 (제30조-제33조)

제 30 조에서는 3 년 이하의 징역 또는 1 천만 원 이하의 벌금에 처하는 기준에 대해서 나타내고 있다. 나열된 6가지 벌칙 중 3가지는 치과기 공사와 안경사에 관한 내용이다. 제 31 조에서는 300 만 원 이하의 벌금 기준에 대해 설명하고 있다. 여기에서도 8 가지 벌칙의 기준을 제시하 고 있는데 한 가지를 제외하고는 모두 치과기공소와 안경업소에 관한 내용이다. 제32조에서는 양벌규정에 대해서 설명하고 있으며 이 또한 감독자와 종업원에 관한 내용으로 엄밀히 따지자면, 개설이 가능한 치과 기공소와 안경업소를 중심으로 한 내용이라 할 수 있다. 제 33 조 에서는 치과 기공소와 안경업소에 관한 과태료에 대한 내용을 나타 내고 있으며, 과태료부과기준에 대해서는 시행령에서 정하고 있다.

\section{3. 일본의 “이학요법사 (물리치료사) 및 작업치료사법”과 한국의 “의료기사 등에 관한 법률”의 비교}

일본의 “이학요법사 (물리치료사) 및 작업치료사법”과 한국의 “의료 기사 등에 관한 법률"의 특징과 공통점 및 차이점은 Table 3 과 같다. 가장 큰 특징이자 차이점은 한국은 6종의 의료기사와 의무기록사, 안경사를 포함하여 8직종이 한 가지 법에 묶여 있으나 일본은 물리 치료사와 작업치료사법으로 단독으로 존재한다는 점이다. 일본의 “이학요법사 (물리치료사) 및 작업치료사법”은 총 6장 22조로 구성되 어 있어 이 법에 대해 이해가 부족한 사람이 보더라도 한눈에 파악할 수 있는 장점이 있다. 일본의 경우 1장 총칙, 2장 면허, 3장 시험, 4 장 업 무, 5 장 물리치료사와 작업치료사 시험위원, 6 장 벌칙으로 구분되어 있으나 한국의 경우에는 장이 없어 내용을 파악하기엔 어려움이 있 다. 좀 더 구체적으로 살펴본다면 장의 구분 없이 총 33 조의 법 조항 중 치과기공소와 안경업소의 개설과 관리에 관한 내용을 제외하면 물리치료사와 작업치료사에 관련된 법 조항은 22조항으로 일본의 동법의 조항과 같은 수이다. 더구나 관련 있는 법 조항조차도 6종의

Table 3. Comparison of the Korean and Japanese Medical Technician Act

\begin{tabular}{|c|c|c|}
\hline & Japan & Korea \\
\hline $\begin{array}{l}\text { Characteristics and } \\
\text { Differences }\end{array}$ & $\begin{array}{l}\text { - Independent Law (Physical Therapist \& Occupational Therapist) } \\
\text { - Chapter 6, Article } 22 \\
\text { - Chapter 1. General Provision, Chapter 2. License, Chapter 3. Examination, } \\
\text { Chapter 4. Duty, Chapter 5. Examination Board for Physical Therapist \& } \\
\text { Occupational Therapist, Chapter 6. Penalty } \\
\text { - Special Acquisition of the License for Foreigner } \\
\text { - After License and period of training courses, eligibility to take the physical } \\
\text { therapist or occupational therapist license each other }\end{array}$ & $\begin{array}{l}\text { - The only one Law, including the optician, medical } \\
\text { recorder and six medical technicians } \\
\text { - No chapter, Article } 33 \\
\text { - Article } 22 \text { is related to Physical therapist \& } \\
\text { Occupational therapist among total Article } 33 \\
\text { - This includes opening and management of Dental } \\
\text { laboratories and eyeglass shops }\end{array}$ \\
\hline Similarity & Composed of Act-Enforcement ordinanc & forcement regulation \\
\hline
\end{tabular}


의료기사와 의무기록사, 안경사를 모두 포함한 8 직종을 다룬 포괄적 법률 조항으로 전문성측면에서 제한점이 있다고 볼 수 있다. 공통점 으로는 법 (법률)-시행령-시행규칙으로 구분되어 있다는 것이다.

\section{결 론}

한국의 경우에 임상병리사, 방사선사, 물리치료사, 작업치료사, 치과 기공사 및 치위생사인 6 가지 종류의 의료기사들과 의무기록사와 안 경사까지 총 8 종류의 직종을 모두 포함하여 “의료기사 등에 관한 법 률”로 묶어 동일한 기준으로 마련된 법이 존재한다. 보건의료기술의 눈부신 발전과 변화가 현실화되고 보건의료분야의 영역이 세분화, 전문화되어 가고 있는 시점에서 8 가지 종류의 직종을 모두 포함하여 하나의 법에 종속시키는 것은 결국 국민의 보건의료서비스에 대한 욕구에 부응하지 못하는 것이라 할 수 있다. $\operatorname{Lim}^{10}$ 은 일본의 방사선 사법에 대한 규정은 팀 의료의 일원으로서 전문직업인으로 양질의 의료를 제공하도록 보장하고 있음을 알수 있다.

일본의 경우엔 “이학요법사 (물리치료사) 및 작업치료사법”이 6장 22조로 나뉘어 법 제정 이전에 충분한 논의를 거쳐 체계적으로 구성 되어 있는 반면 한국의 경우엔 장의 구분이 없고 총 33 조로 이루어져 있으며 이 조차도 치과기공소와 안경업소에 대한 내용이 11 가지나 된 다. 이러한 결과의 이유는 한국의 경우 법률의 제정과 개정이 보건의 료계의 중요한 이슈와 사안에 따라 추가되어 나타나는 형태라고 할 수 있다. Kim 등 11,12 은 일본의 경우 의료인은 의료법과 기타 인력은 각 개별 법에서 각 종별에 적합한 형태의 자격과 면허, 시험, 업무 등을 규 정하고 있으나 한국의 경우 의료법에서조차 의사에 관한 규정이 주를 이루며 기타 인력들의 내용에 명확한 기준과 체계를 갖고 있지 않다 고 지적하고 있다. 해당 의료기사의 명확한 이해 없이 위원회가 조직 되고 법률이 만들어지다 보니 이러한 결과가 발생한다고 할수 있다.

한국의 경우 “의료기사”란 의사 또는 치과의사의 지도 아래 진료 나 의화학적 검사에 종사하는 사람으로 규정하고 있으나, 일본은 각 의료기사에 따라 다르게 규정하고 있으며 구체적으로 살펴보면, 이 학요법사 (물리치료사)나 작업치료사의 경우 의사의 지시하에 이학 요법과 작업요법을 업으로 하는 자로 규정하고 있다. $\mathrm{Yang}^{13}$ 은 일본의 경우 의사와 의료기사 간의 관계를 규정하는 용어에서 해당 의료기 사의 업무에 관한 성격을 엿볼 수 있으며 이러한 관계의 정도에 따라 각 법률에서 규정하는 바가 달라진다고 해석하였다. Lee와 Park ${ }^{14}$ 은 '의료지도'라는 용어보다 '의사가 발행한 의뢰서 또는 처방에 의하여 업무를 행하여야 한다.로 개정하는 것이 타당하다고 보고하고 있다. Baek와 Yang ${ }^{15}$ 은 “지도아래”의 규정을 해석함에 있어서 개설이 가능 한 치과기공소 및 안경업소에 근무하는 치과기공사나 안경사와 의료 기관에 고용되어 근무하는 물리치료사나 작업치료사에게 같은 조
항을 동일하게 적용하는 것은 모순이라고 지적한다. 특히 재활치료 는 팀 접근으로 이루어지므로 의사와의 관계가 수평적인 관계로 법 에서도 규정되어야 할 것이다. 흔히 의사 직군이 의료기사를 지도로 두어야 한다는 이유는 응급상황의 대처, 경제적 이점, 환자의 편리성 및 만족도 등이 높다는 데 있다. 그러나 재활치료에 있어서 응급상황 이란 거의 존재하지 않고, 발생한다 하더라고 응급의료시스템에 의 해 환자를 이송하면 된다. 의사를 고용하는 것보다 상대적으로 저비 용의 물리치료사가 치료하는 것이 경제적일 수 있으며, 환자측면에서 도 그 요구도에 따라 편리성이나 만족도가 높으므로 재활치료에 있 어 수평적인 관계의 정립은 필요하다.

본 연구를 통하여, 다음의 몇 가지 결론을 얻을 수 있었다.

1. 일본은 “이학요법사 (물리치료사) 및 작업치료사법”으로 개별 법 이 존재하나 한국의 “의료기사 등에 관한 법률”은 8 가지 종류의 직종 을 모두 포함하여 동일한 기준을 적용하고 있다.

2. 일본의 “이학요법사 (물리치료사)법 및 작업치료사법”은 총 6장 으로 구분되어 내용파악도 쉽고 법 개정이 용이하나, 한국의 “의료기 사 등에 관한 법률"은 장의 구분이 없고 보건의료 이슈에 따라 필요 한 조항을 추가하는 형태로 발전되어 왔다.

3. “의료기사 등에 관한 법률”은 “의료법”과의 관계를 생각하며 개 정되어야 하며, 법 개정 이전에 의사와 의료기사의 보다 수평적 관계 발전과 정립이 필요하다.

마지막으로, 본 연구를 통하여 급변하는 보건의료환경과 보건의 료기술의 전문화 및 분업화 속에서 “의료기사 등에 관한 법률”로 8 개 의 모든 직종을 동일한 기준으로 적용하기에는 논리적으로나 현실적 으로 모순이 있음을 알 수 있었다. 하지만 법이라는 것은 역사이며 그 사회의 실정을 담고 있다고 할 수 있으므로, 향후 동법의 제정 및 개 정에 있어서 역사적인 맥락을 함께 파악하고, 나아가 물리치료사와 작업치료사뿐만 아니라, 8 개 직군의 개별 법이 존재하는 일본의 경우 를 연구하여, 그 밖의 모든 의료기사와 관련된 법의 나아가야 할 방 향을 설정해야할 것이다.

\section{ACKNOWLEDGEMENTS}

This work was supported by Dongseo University, "Dongseo Frontier Project" Research Fund of 2014

\section{REFERENCES}

1. Ko KW, Lee KW, Seo SK. A study on the relation between physical therapist professionalism and organizational and job characteristics. J Kor Phys Ther. 2013;25(5):343-51.

2. Kim YS, Yoon BC. Conflict of interest groups on the health insurance 
policy deliberation committee affect the medical insurance cost of physical therapy. J Kor Phys Ther. 2013;25(2):43-48.

3. Kim DH, Shon MJ. The effects of soci-demographic characteristics of physical therapist for the awareness of necessity of opening independent clinic \& professionalism of the physical therapist. IJOC. 2015;15(12): 378-86.

4. Bae SS, Kim DY, Nam SW et al. The legal system for the independent practice of physical therapy. J Kor Phys Ther. 1998;10(1):253-63.

5. Korean Ministry of Government Legislation. Medical technicians. ETC. ACT. Seoul, 2015.3.

6. Health focus. 300,000 rehabilitation therapists challenge to the medical community. Seoul, 2015.12.

7. Lee HJ, Song JM. Development of revised Korean Version of ICF. J Kor Phys Ther. 2014;26(5):344-50.

8. Japanese Ministry of Health, Labor and Welfare. Physical therapist and occupational therapist ACT. Tokyo, 2015.11.

9. Lee HY. Medical system and law in Japan. The S on the Law and Policy.
2003;3(1):51-75.

10. Lim CS. A study on the issues and improving directions of the rules related radiologic technologist in medical law. Kor Soc Raio Science. 1994; 17(1):87-96.

11. Kim KH, Sohn MS, Park HW et al. A comparison of the Korean and Japanese medical law system. J Prev Med Public Health. 2001;53. Fall Conference.

12. Kim KH, Won SA, Sohn MS. A study on the medical law systems in Korea and Japan. Kor J of Medicine and Law. 2001;9(2):99-127.

13. Yang IK. Quantitative comparative study on the medical technician between Japan and Korea. J of the Korean Hosp Association. 1994;(11):2030 .

14. Lee JW, Park EG. A study on the existing law and direction for revision in the physical therapist. Kor Law Association. 2010;38:393-412.

15. Baek JC, Yang SH. Problems of the physical therapy system of Korea and their solutions. The Kor Enter Industry Association. 2011;5(4):138-46. 